\title{
DETERMINAÇÃO dA EVAPOTRANSPIRAÇÃO POTENCIAL DO BRASIL APLICANDO O MODELO DE THORNTHWAITE A UM SISTEMA DE INFORMAÇÃO GEOGRÁFICA
}

\author{
Marcelo Cid de Amorim, Luciana Rossato e Javier Tomasella \\ Instituto Nacional de Pesquisas Espaciais - Centro de Previsão de Tempo e Estudos Climáticos (INPE/CPTEC) \\ Rod. Pres. Dutra, km 40 - CEP 12630-000 Cachoeira Paulista, SP - Fone (12) 560-8518 - FAX (12) 561-2835 \\ amorim@cptec.inpe.br, rossato@cptec.inpe.br e javier@cptec.inpe.br
}

\section{RESUMO}

Os dados de temperatura média do ar registrados nas normais climatológicas (1961 - 1990) do INMET/MA foram aplicados ao modelo de Thornthwaite para estimar a evapotranspiração potencial mensal no Brasil, associado a um Sistema de Informação Geográfica (SIG). Para corrigir a tendência desse modelo em superestimar a $E T_{p}$ quando a temperatura média do ar é maior que $26,5^{\circ} \mathrm{C}$, foi introduzido um fator de correção $\left(C_{T}\right)$ que simplificou o seu uso em operações computacionais, como um Sistema de Informação Geográfica. Dessa forma, os resultados foram imagens de fácil interpretação e de grande valor climatológico.

\section{INTRODUÇÃO}

O interesse dos povos pelo conhecimento da taxa de evaporação (E) e/ou evapotranspiração (ET), vem de períodos remotos. Brutsaert (1982) faz referência aos antigos gregos, no período romano e na Idade Média. Entretanto, é consenso entre os pesquisadores de que se deu um importante passo, com o surgimento dos modelos matemáticos, para estimar os processos de evaporação e evapotranspiração.

Warren Thornthwaite, em 1948, propôs um modelo simples para estimar a evapotranspiração potencial $\left(\mathrm{ET}_{\mathrm{p}}\right)$, baseado em dados de temperatura média do ar e do fotoperíodo de áreas secas dos Estados Unidos. Este modelo apresenta fortes aplicações geográficas, sendo de grande valor prático na estimativa da evapotranspiração em áreas onde não há disponibilidade de outros dados além dos de temperatura. Sua maior utilização, contudo, é para estimativa do balanço hídrico, cujos resultados têm sido largamente usados, como parâmetros, para estabelecer comparações entre condições climáticas reinantes em áreas distintas, com vistas à realização de zoneamento agroclimático. Do ponto de vista da climatologia agrícola, o modelo de Thornthwaite associado a um Sistema de Informação Geográfica (SIG) vem a ser uma poderosa ferramenta para os estudos do balanço hídrico e da evapotranspiração regional de extensas áreas.

Um dos grandes problemas com que se defrontam os técnicos de irrigação e drenagem, ou aqueles que trabalham com as interações soloágua-planta-atmosfera, é a contabilização da água perdida por evaporação no balanço hídrico do solo. A taxa de água perdida pela superfície, por evaporação $(E)$ e/ou, transpiração $(T)$, é de grande importância na determinação das necessidades hídricas dos cultivos agrícolas de qualquer região. Dessa forma, é possível fazer um monitoramento eficiente dos recursos hídricos e gerar parâmetros que tornem o manejo agrícola uma atividade lucrativa e ecologicamente viável.

Assim, o objetivo deste trabalho foi desenvolver uma metodologia para determinar $\mathrm{ET}_{\mathrm{p}} \mathrm{em}$ um ambiente georreferenciado, em escala mensal, para todo o território brasileiro. Foram utilizadas séries históricas dos dados de temperatura média do ar, registrados nas Normais Climatológicas, como também dos dados recentes, de temperatura média do ar, provenientes das estações meteorológicas do INMET/MA distribuídas no Brasil, aplicando-as ao modelo de Thornthwaite (1948) associado a um Sistema de Informação Geográfica (SIG).

\section{MATERIAL E MÉTODOS}

Neste estudo, foram utilizados dados de temperatura média do ar do Brasil referentes ao ano de 1997, como também os dados históricos de temperatura média mensal do ar registrados nas Normais Climatológicas (1961 - 1990), do Instituto Nacional de Meteorologia (INMET/MA). Nesta publicação (Brasil, 1992), são encontradas aproximadamente 206 estações meteorológicas, espalhadas pelas cinco grandes regiões da federação brasileira (Norte, Nordeste, Centro-Oeste, Sul e Sudeste), situadas em todas as Unidades Federais (Figura 1). 


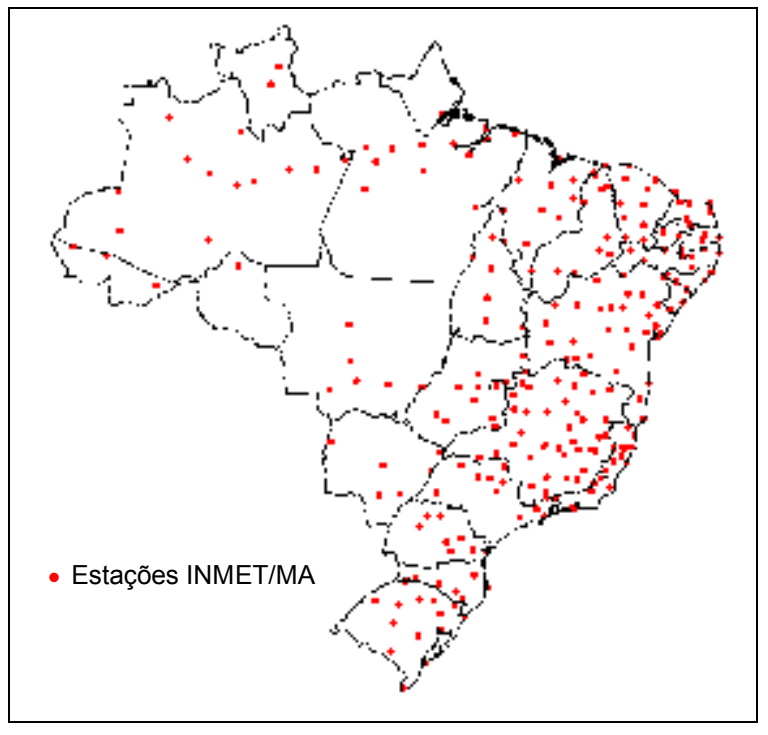

Figura 1. Distribuição espacial das estações meteorológicas do INEMET/MA em todo o território nacional (dados parciais).

Para determinar a evapotranspiração potencial de cada mês $(j=1,2,3, \ldots, 12)$, foi utilizado o modelo de Thornthwaite (1948), apresentando a seguinte notação matemática

$$
E T_{p}=E_{j} \cdot C_{j}
$$

em que $\mathrm{ET}_{\mathrm{p}}$ é a evapotranspiração potencial acumulada, mm/mês; $E_{j}$ a evapotranspiração potencial não ajustada, mm/mês e $C_{j}$ o fator de correção que leva em consideração o fotoperíodo médio e o número de dias do mês em questão.

\section{Evapotranspiração potencial não- ajustada $\left(E_{j}\right)$}

Ej representa a evapotranspiração estimada para um mês-padrão de 30 dias e com duração do período diurno de 12 horas. Quando o valor médio da temperatura do ar for inferior a $26,5^{\circ} \mathrm{C}$ $\left(t_{j}<26,5^{\circ} \mathrm{C}\right)$, Thornthwaite (1948) propôs estimar Ej usando o seguinte modelo

$$
E_{j}=16\left(10 \frac{t_{j}}{l}\right)^{a}
$$

sendo $t_{j}$ a temperatura média mensal $\left({ }^{\circ} \mathrm{C}\right)$ e I o índice térmico anual, dado por:

$$
I=\sum_{j=1}^{12}\left(\frac{t_{j}}{5}\right)^{1,514}
$$

a que é função do I, determinada por:

$$
\begin{gathered}
a=6,75 \cdot 10^{-7} \cdot I^{3}-7,71 \cdot 10^{-5} \cdot .^{2}+ \\
1,792 \cdot 10^{-2} \cdot I+0,49239
\end{gathered}
$$

Para temperatura igual ou superior a $26,5{ }^{\circ} \mathrm{C}\left(\mathrm{t}_{\mathrm{j}} \geq 26,5^{\circ} \mathrm{C}\right)$, Thornthwaite propôs o uso da Tabela 1; dessa forma, corrige-se a tendência da Equação (2) em superestimar $\mathrm{ET}_{\mathrm{p}}$.

\section{Fator de correção $\left(C_{j}\right)$}

O fator de correção $C_{j}$ leva em consideração o fotoperíodo médio e a latitude do local, bem como o número de dias do mês em estudo. $A$ importância desse coeficiente no cálculo da $E_{\mathrm{p}}$ foi descrita por Varejão \& Reis (1990) e Vianello \& Alves (1991). Esta correção é dada pela seguinte equação:

$$
C_{j}=\frac{N_{j}}{12 \cdot D_{j}}
$$

em que $D_{\mathrm{j}}$ é o número de dias do mês $\mathrm{j}$ (em janeiro, $D_{1}=31$; em fevereiro, $D_{2}=28$; etc.) e $N_{j}$ o fotoperíodo do dia 15 , considerado representativo da média desse mês.

$$
\mathrm{N}_{\mathrm{j}}=2 \cdot \frac{[\operatorname{arc} \cdot \cos (-\operatorname{tg} \Phi \cdot \operatorname{tg} \delta+0,83)]}{15}
$$

sendo $\Phi$ a latitude (negativa para hemisfério sul) do local e $\delta$ a declinação do sol estimada pela equação proposta por Klein (1977):

$$
\delta=23,45^{\circ} \cdot \operatorname{sen}\left[360^{\circ} \cdot\left(\frac{284+D_{j}}{365}\right)\right]
$$

\section{Fator de correção da temperatura $\left(C_{T}\right)$}

Como a utilização da Equação (2) está limitada aos valores inferiores a $26,5^{\circ} \mathrm{C}$, sua aplicação no SIG é complexa para períodos de temperaturas médias igual ou superior a $26,5^{\circ} \mathrm{C}$. Nesse caso, é necessário aplicar critérios de classificação do plano das temperaturas, a fim de sepa- 
Tabela 1. Valores da evapotranspiração não-corrigida $\left(E_{j}\right), \mathbf{m m} / \mathbf{m e ̂ s}$, para temperaturas do ar $\left(t_{i}\right)$ igual ou superior a $26,5^{\circ} \mathrm{C}\left(\mathrm{t}_{\mathrm{i}} \geq 26,5^{\circ} \mathrm{C}\right)$.

\begin{tabular}{ccccccccccc}
\hline & \multicolumn{10}{c}{ Décimos de grau } \\
$\mathrm{t}_{\mathbf{l}}$ & 0,0 & 0,1 & 0,2 & 0,3 & 0,4 & 0,5 & 0,6 & 0,7 & 0,8 & 0,9 \\
\hline 26 & - & - & - & - & - & 135,0 & 135,0 & 138,0 & 138,0 & 138,0 \\
27 & 138,0 & 141,0 & 141,0 & 141,0 & 144,0 & 144,0 & 144,0 & 144,0 & 147,0 & 147,0 \\
28 & 147,0 & 150,0 & 150,0 & 150,0 & 150,0 & 153,0 & 153,0 & 153,0 & 153,0 & 156,0 \\
29 & 156,0 & 156,0 & 156,0 & 156,0 & 159,0 & 159,0 & 159,0 & 159,0 & 162,0 & 162,0 \\
30 & 162,0 & 162,0 & 162,0 & 165,0 & 165,0 & 165,0 & 165,0 & 165,0 & 168,0 & 168,0 \\
31 & 168,0 & 168,0 & 168,0 & 171,0 & 171,0 & 171,0 & 171,0 & 171,0 & 171,0 & 174,0 \\
32 & 174,0 & 174,0 & 174,0 & 174,0 & 174,0 & 174,0 & 177,0 & 177,0 & 177,0 & 177,0 \\
33 & 177,0 & 177,0 & 177,0 & 177,0 & 180,0 & 180,0 & 180,0 & 180,0 & 180,0 & 180,0 \\
34 & 180,0 & 180,0 & 180,0 & 183,0 & 183,0 & 183,0 & 183,0 & 183,0 & 183,0 & 183,0 \\
35 & 183,0 & 183,0 & 183,0 & 183,0 & 183,0 & 183,0 & 183,0 & 183,0 & 183,0 & 183,0 \\
36 & 183,0 & 183,0 & 186,0 & 186,0 & 186,0 & 186,0 & 186,0 & 186,0 & 186,0 & 186,0 \\
37 & 186,0 & 186,0 & 186,0 & 186,0 & 186,0 & 186,0 & 186,0 & 186,0 & 186,0 & 186,0 \\
\hline
\end{tabular}

rar as regiões em função dos intervalos de temperaturas propostos por Thornthwaite, ou seja, regiões para Equação (2) quando $t_{j}<26,5^{\circ} \mathrm{C}$ e regiões para os valores da Tabela 1 quando $\mathrm{t}_{\mathrm{j}} \geq 26,5^{\circ} \mathrm{C}$.

Com a finalidade de simplificar o processo de cálculo, foi utilizado na Equação (2) um fator de correção de temperatura, denominado $\mathrm{C}_{\mathrm{T}}$ proposto por Amorim et al. (s. d.). O coeficiente $\mathrm{C}_{\mathrm{T}}$ produz uma atenuação do valor de $\mathrm{E}_{\mathrm{j}}$ estimado pela Equação (2), de maneira a igualar os valores fornecidos pela Tabela 1 quando a temperatura for superior a $26,5^{\circ} \mathrm{C}$, caso contrário, $\mathrm{C}_{\mathrm{T}}$ tenderá a 1 . Assim, otimiza-se o modelo de Thornthwaite diminuindo a tendência de superestimar a evapotranspiração potencial para regiões que apresentam altas temperaturas.

Para determinar a variação do coeficiente $\mathrm{C}_{\mathrm{T}}$ com a temperatura, foram calculados os valores de $E_{j}$ utilizando a Equação (2) para temperaturas superiores a $26,5^{\circ} \mathrm{C}$ com os dados das 206 estações da Figura 1. Também foram estimados os valores de $\mathrm{ET}_{\mathrm{p}}$ para as mesmas temperaturas $\mathrm{u}$ sando-se a Tabela 1. A Figura 2 mostra o gráfico da variação do coeficiente $C_{T}$, que foi plotado em função da temperatura. Para extrapolar o intervalo de observação, foram incluídas temperaturas de até $40{ }^{\circ} \mathrm{C}$.

O fator de correção pode ser representado analiticamente por uma função do tipo:

$$
C_{T}=1-e^{-e^{-\alpha\left(t_{j}-\beta\right)}}
$$

sendo $\alpha$ e $\beta$ parâmetros de ajuste.

Os valores de $\alpha$ e $\beta$ que melhor ajustam o coeficiente $C_{T}$ são, respectivamente, 0,28 e 31,1.

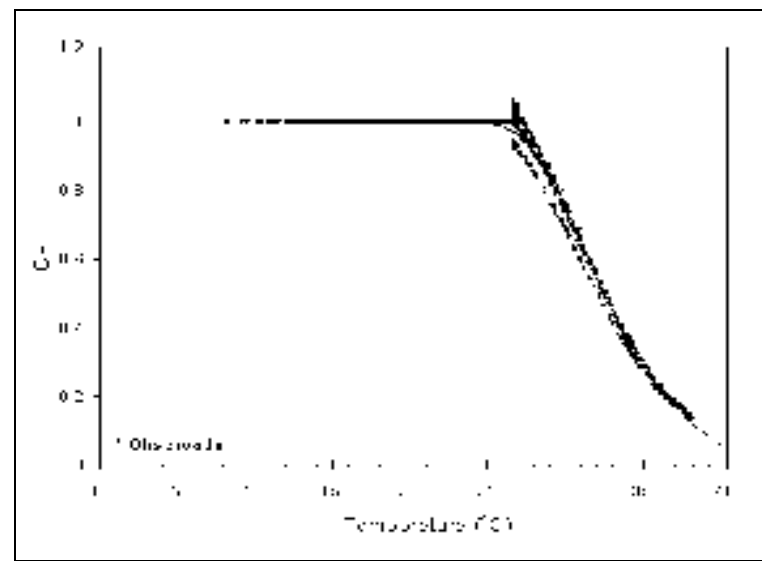

Figura 2. Fator de correção da temperatura $\left(\mathrm{C}_{\mathrm{T}}\right)$.

Com esta correção, o modelo de Thornthwaite fica adaptado para qualquer condição de clima e tempo, apresentando maiores facilidades para efetuar cálculos e operações computacionais, por evitar o uso de tabelas ou de outras equações de correção. Com os devidos ajustes do coeficiente $\mathrm{C}_{\mathrm{T}}$, reescreve-se a equação de Thornthwaite da seguinte forma:

$$
E_{j}=16\left(10 \frac{t_{j}}{\mathrm{l}}\right)^{a} \cdot\left[1-e^{-e^{-0.28 \cdot\left(t_{j}-31.1\right)}}\right]
$$

Os recursos da matemática boleana inerente a um Sistema de Informação Geográfica produzirão mapas interpolados da evapotranspiração potencial para cada mês, no Brasil, da seguinte forma. Primeiramente, serão gerados mapas de 
cada variável envolvida no cálculo da evapotranspiração, ou seja, mapas do fator de correção mensal $\left(C_{j}\right)$ e dos coeficientes do modelo de Thornthwaite (mapas do índice térmico I e do expoente a; esses valores foram calculados a partir dos dados das Normais Climatológicas de temperatura média do ar de cada mês; dessa forma, têm-se mapas substancialmente climáticos). Finalmente, serão produzidos mapas mensais referente à temperatura média do ar $\left(\mathrm{t}_{\mathrm{i}}\right)$ no Brasil a partir de dados do ano de 1997 fornecidos pelo INEMET (veja no fluxograma proposto na Figura 3).

\section{RESULTADOS E DISCUSSÃO}

As Figuras 4 a 7 referem-se aos mapas da evapotranspiração potencial $\left(E_{\mathrm{p}}\right)$ sazonal de todo o território brasileiro, calculados a partir da média aritmética das evapotranspirações dos meses referente as estações do ano no hemisfério sul. Os resultados mostraram-se coerentes com as características climáticas de cada região geográfica do Brasil, sendo os maiores valores de $\mathrm{ET}_{\mathrm{p}}$ observados nas regiões Centro-Oeste, Nordeste e Norte e os menores, no Sul e Sudeste. No processamento dos dados de temperatura para gerar essas imagens, o Sistema de Informação Geográfica (SIG) mostrou-se uma ferramenta eficiente, simples e de fácil interpretação, apresentando ainda como vantagens o baixo custo no processamento dos dados e o fato de gerar mapas significativamente confiáveis.

De acordo com resultados observados, o uso do modelo de Thornthwaite na sua forma geral (Equação 2), aplicado a grandes áreas como o Brasil, causam erros, principalmente se a temperatura média do ar for igual ou superior a $26,5^{\circ} \mathrm{C}$. Este modelo apresenta tendência natural em superestimar a evapotranspiração potencial; assim, foi observado que, durante alguns meses do ano, em uma grande área no Brasil, coincidindo principalmente com as regiões Norte, Nordeste e CentroOeste, a temperatura média do ar foi superior a $26,5{ }^{\circ} \mathrm{C}$. Dessa forma, a introdução do coeficiente $\mathrm{C}_{\mathrm{T}}$ para eliminar a tendência de superestimação foi uma medida necessária, sendo eficiente e prática para realizar esta correção. Outro fator que não pode ser esquecido no cálculo é o fator de correção $\left(C_{\mathrm{j}}\right)$ que leva em consideração o fotoperíodo médio, número de dias do mês em questão e da latitude do local.

A energia proveniente do sol é fonte primária em todos os processos evaporativos que ocorrem no sistema solo-água-planta-atmosfera; desprezá-la nos cálculos de modelagens e nos estudos agroclimáticos seria um grave erro. Um exemplo: sendo esta correção não considerada em alguns estados de maior latitude sul, como o Rio Grande do Sul, a perda e o excesso na demanda evaporativa ultrapassariam as taxas de $20 \%$ no período a ser determinado, em razão de o número do brilho solar para os meses entre novembro e fevereiro apresentar médias superiores a 14 horas e, para os meses entre abril e agosto, inferior a 10 horas.

O método de Thornthwaite tem sido bastante criticado por utilizar apenas dados de temperatura do ar como variável independente. De acordo com Pereira \& Camargo (1989), essa crítica é injusta, pois inúmeros outros métodos também utilizam apenas a temperatura como condicionante da evapotranspiração. Esses autores concluíram ainda que este método é apropriado para estimativa da evapotranspiração potencial, se as condições micrometeorológicas forem bem definidas; sua utilização não é recomendada para regiões ou locais que estão sujeitos ao fenômeno da advecção, ou seja, às condições de oásis.

A aplicação do geoprocessamento para obter informações espaciais de elementos climáticos tornou-se uma poderosa ferramenta, pois, além da simplicidade na forma com que se aplicam os dados, as respostas são significativamente confiáveis. Com este estudo, abre-se imensa possibilidade de desenvolvimento de outros trabalhos, como na determinação de evapotranspiração de referência diária $\left(\mathrm{mmd}^{-1}\right)$ utilizando modelos mais sensíveis às condições ambientais (Blaney-Criddle - FAO, Penman-Monteith - FAO, Penman original, etc.), em caracterizações físico-hídricas dos solos, manejo de culturas agrícolas, viabilidade econômica de projetos hidro-agrícolas, avaliação ecológica, etc.

\section{CONCLUSÕES}

O uso do modelo de Thornthwaite é puramente geográfico. Dessa forma, é recomendado que esse tipo de estudo seja aplicado para fins climáticos, como na caracterização de grandes áreas na forma de zoneamento agroclimático. Ele não deve ser adotado para determinados fins agrícolas quando não se conhecem as condições micrometeorológicas do local.

$\mathrm{O}$ emprego do fator de correção $\left(\mathrm{C}_{\mathrm{j}}\right)$, que leva em consideração o fotoperíodo médio, número de dias do mês em questão e da latitude do local, é imprescindível no cálculo do modelo de Thornthwaite para estimar a evapotranspiração potencial para áreas extensas. 


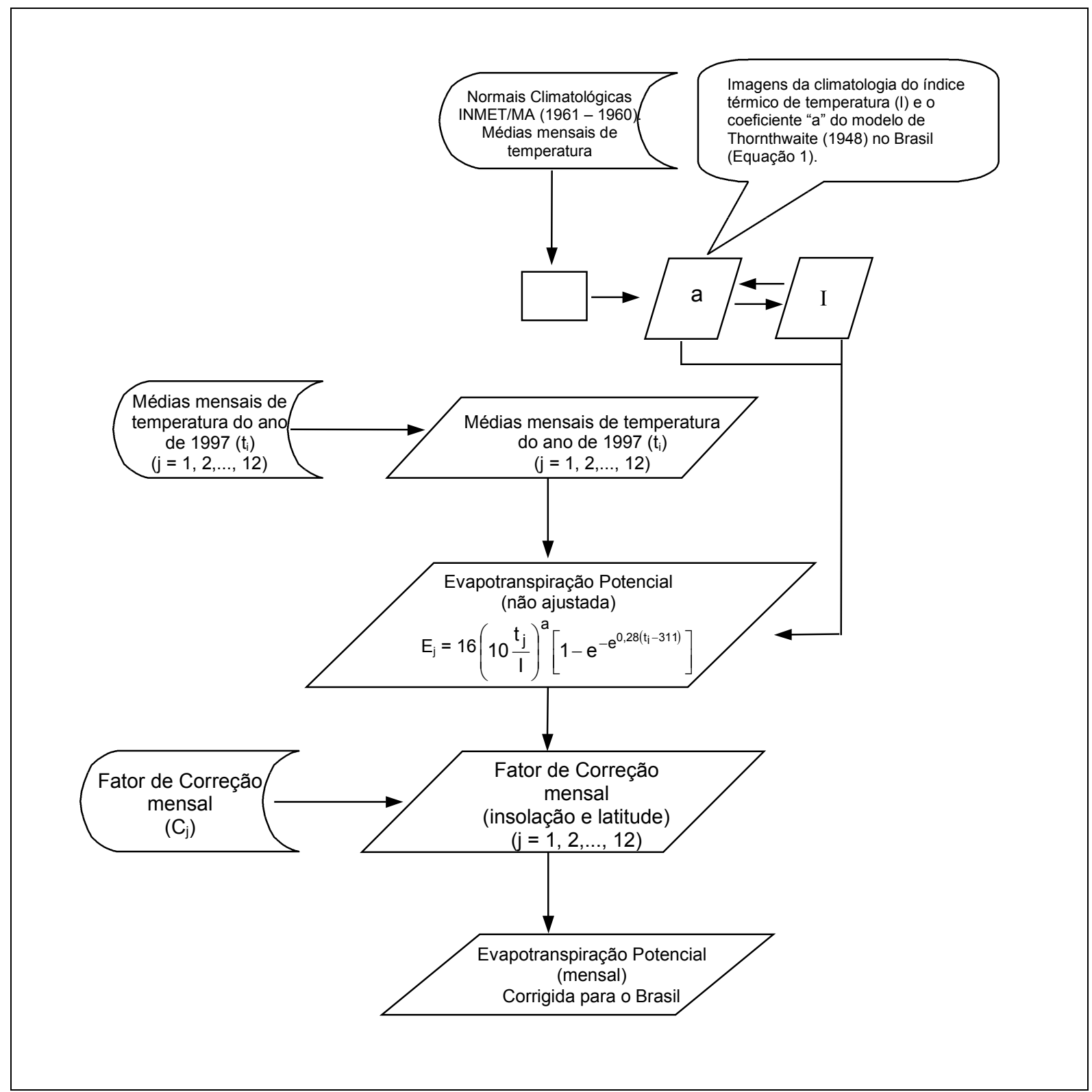

Figura 3. Fluxograma aplicado a um Sistema de Informação Geográfica, para obtenção dos mapas de evapotranspiração potencial mensal no Brasil.

A introdução do fator de correção $C_{T}$ no modelo de Thornthwaite é uma alternativa válida e eficiente, pois, além de corrigir a tendência de superestimar a $\mathrm{ET}_{\mathrm{p}}$, simplificou o uso deste modelo em operações computacionais, como em um Sistema de Informação Geográfica.

O uso de um Sistema de Informação Geográfica para espacialização dos dados climáticos, seja medido ou estimado por modelos matemáti- cos, é uma alternativa simples, de baixo custo, e as respostas são significativamente confiáveis.

\section{AGRADECIMENTOS}

Os autores agradecem ao CNPq, pelo apoio financeiro; ao Instituto Nacional de Pesquisas Espaciais / Centro de Previsão de Tempo e Estudos Climáticos (INPE/CPTEC) que, com seu apoio, proporcio- 


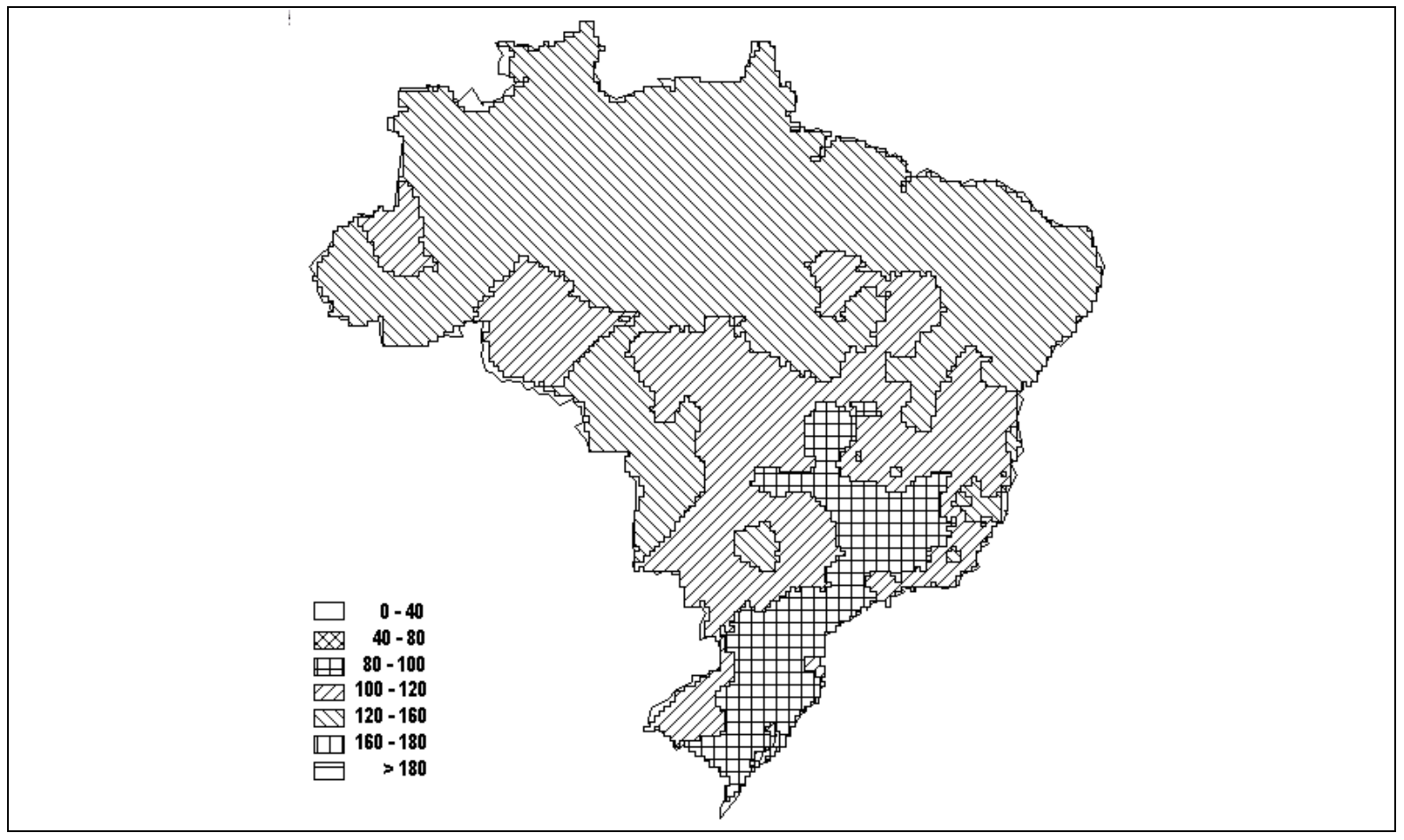

Figura 4. Evapotranspiração potencial, $\mathrm{mm} / \mathrm{mês,} \mathrm{referente} \mathrm{aos} \mathrm{meses} \mathrm{de} \mathrm{dezembro,} \mathrm{janeiro} \mathrm{e}$ fevereiro (verão - 1997).

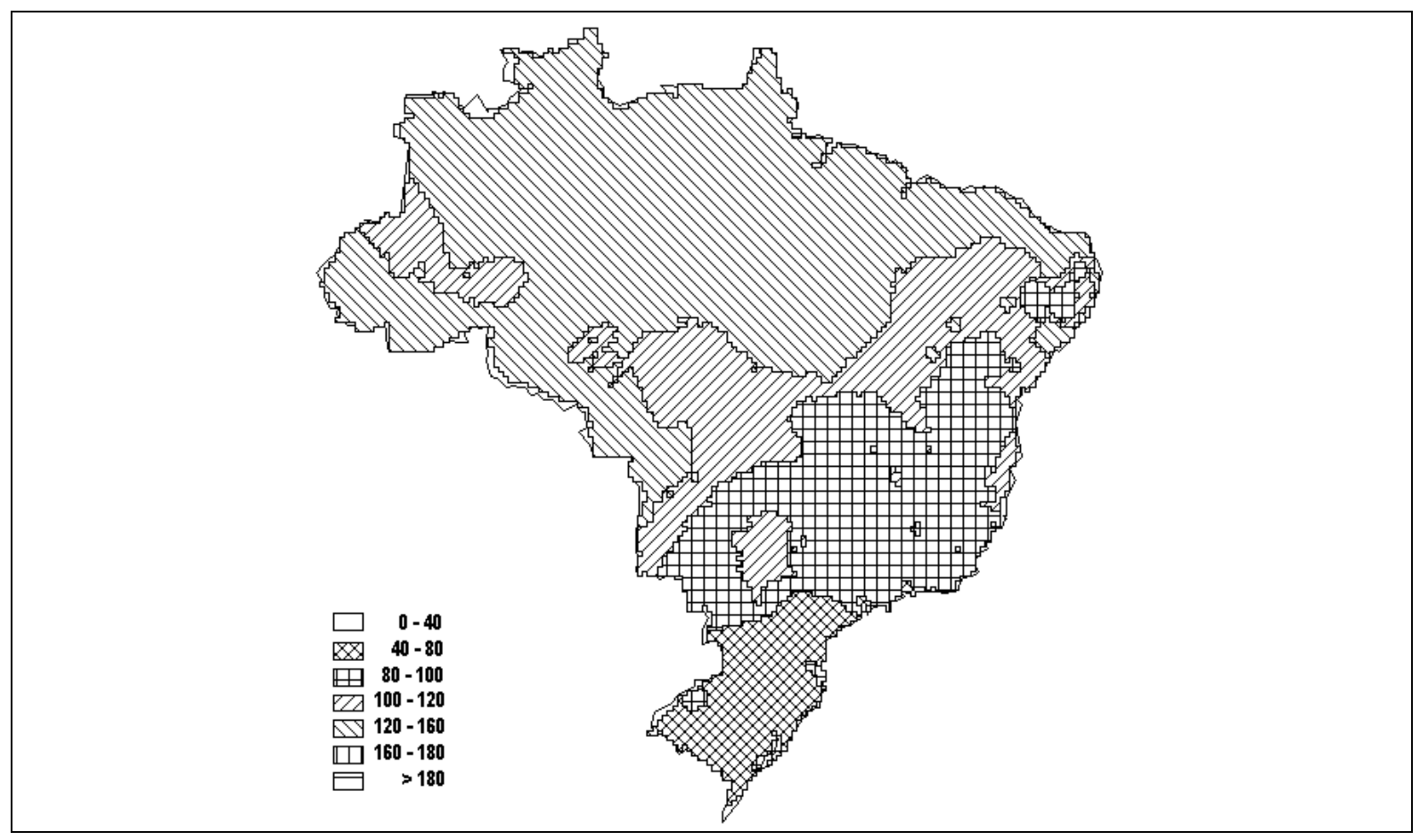

Figura 5. Evapotranspiração potencial, $\mathrm{mm} / \mathrm{mês,} \mathrm{referente} \mathrm{aos} \mathrm{meses} \mathrm{de} \mathrm{março,} \mathrm{abril} \mathrm{e} \mathrm{maio}$ (outono - 1997). 


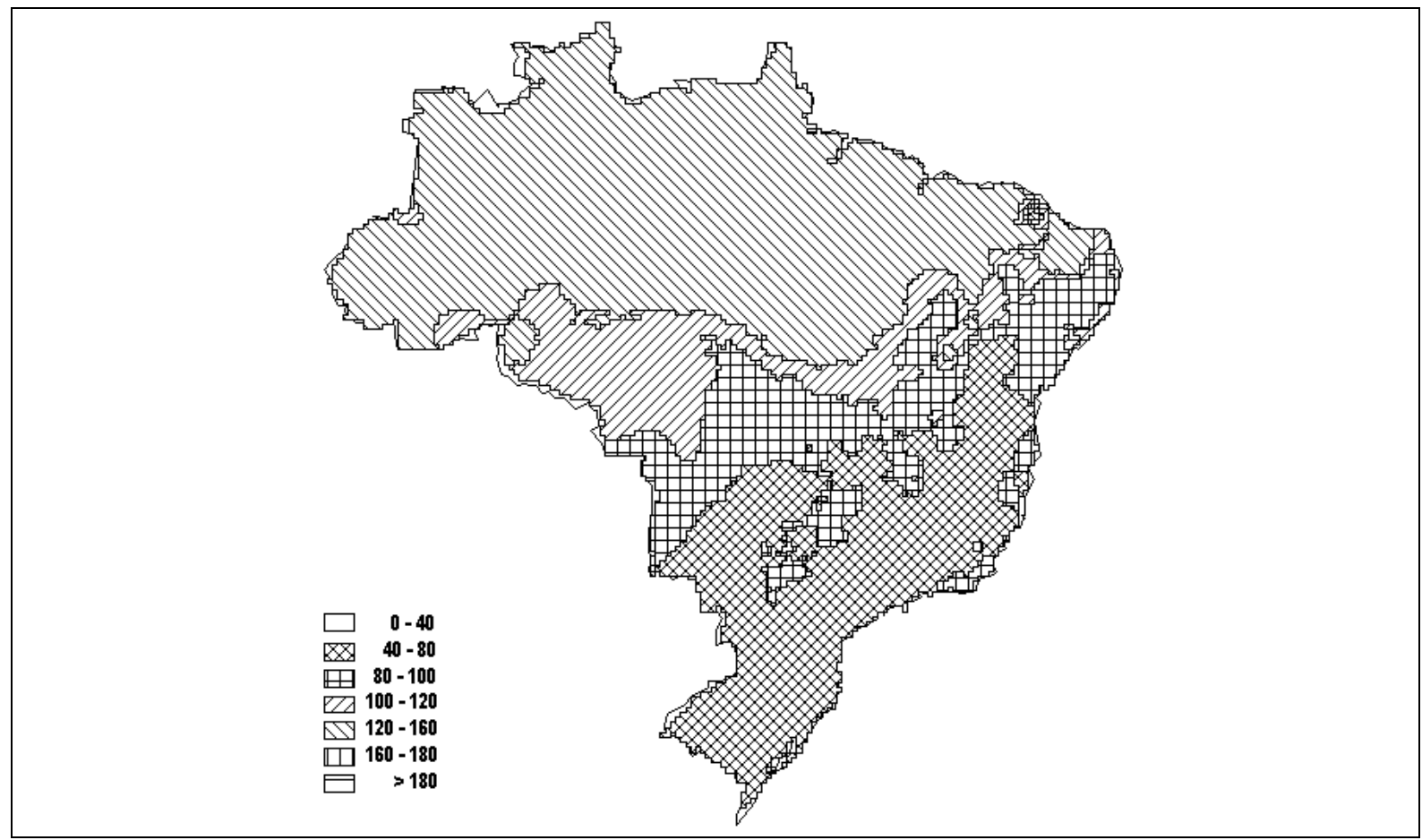

Figura 6. Evapotranspiração potencial, $\mathrm{mm} / \mathrm{mês,} \mathrm{referente} \mathrm{aos} \mathrm{meses} \mathrm{de} \mathrm{junho,} \mathrm{julho} \mathrm{e} \mathrm{agosto}$ (inverno - 1997).

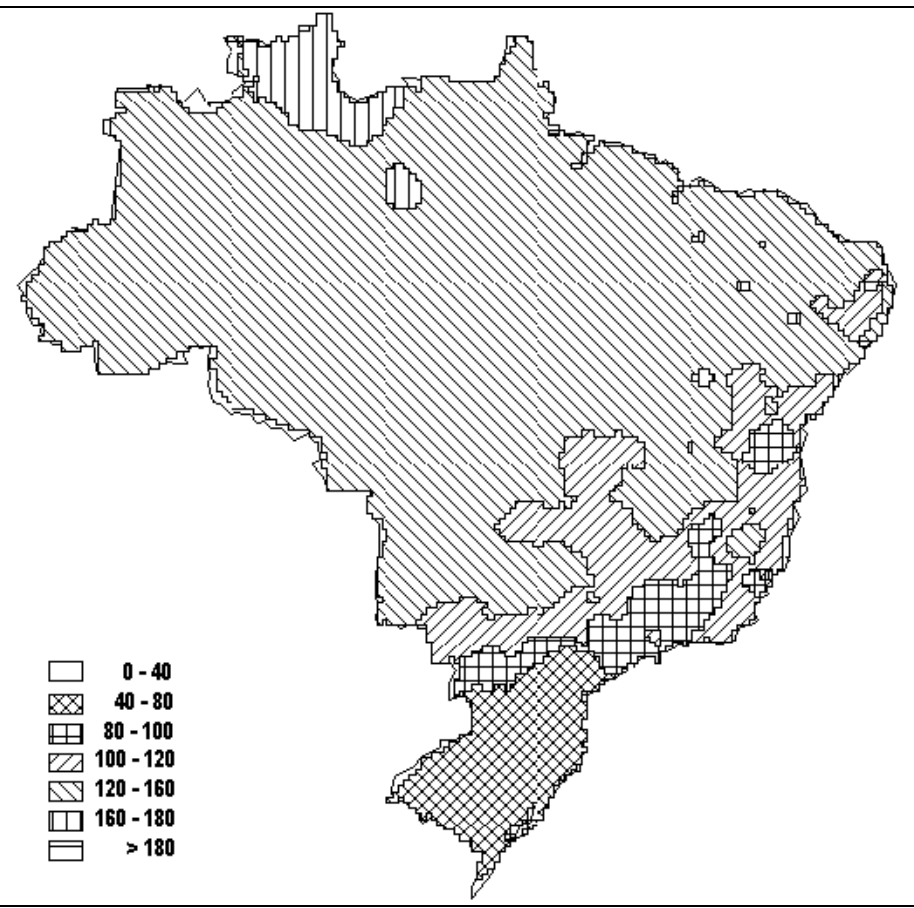

Figura 7. Evapotranspiração potencial, $\mathrm{mm} / \mathrm{mês,} \mathrm{referente} \mathrm{aos} \mathrm{meses} \mathrm{de} \mathrm{setembro,} \mathrm{outubro} \mathrm{e}$ novembro (primavera - 1997). 
naram a estrutura necessária à elaboração deste trabalho e; à equipe da revista CLIMANÁLISE, pelo fornecimento dos dados. Agradecem ainda às seguintes entidades, pelo valoroso intercâmbio de informações: Instituto Nacional de Meteorologia (INMET/MA), Departamento de Engenharia Agrícola da Universidade Federal de Viçosa (DEA-UFV) e Instituto de Pesquisas Hidráulicas da Universidade Federal do Rio Grande do Sul (IPH/UFRGS).

\section{REFERÊNCIAS}

AMORIM, M. C., TOMASELLA, J., SEDIYAMA, G. C., ROSSATO, L. (s. d). A factor of correction to the Thornthwaite model. Revista Brasileira de Agrometeorologia-SBA, Santa Maria, RS (em revisão)

BRASIL (1992). Ministério da Agricultura e Reforma Agrária. Normais Climatológicas (1961 1990). Brasília: EMBRAPA/SPI, p84.

BRUTSAERT, W. H. (1982). Evaporation into the atmosphere. Dordrecht, Holland: D. Reidel, p299.

KLEIN, S. A. (1977). Calculation of monthly average insolation on titled surfaces. Solar Energy, v.19. p325-329.

PEREIRA, A. R., CAMARGO, A. P. (1989). An analisys of the criticism of Thornthwaite's equation for estimating potential evapotranspiration. Agriculture Forest Meteorological, v.46, p149-157.

THORNTHWAITE, W. C. (1948). An approach toward a rational classification of climate. Geographical Review, v.38, p55-94.

VAREJÃO-SILVA, M. A., REIS, A. C. S. (1990). Meteorologia. Recife (PE), p461 (no prelo)

VIANELLO, R. L., ALVES, A. R. (1991). Meteorologia básica e aplicações. Viçosa, MG: Universidade Federal de Viçosa, p449.

\section{Determination of Monthly Potential Evapotranspiration in Brazil Applying the Thornthwaite Model to a Geographic Information System}

\section{ABSTRACT}

Using mean air temperature data recorded in the Normais Climatológicas (1961 - 1990) of INMET/MA, we apply these data to the Thornthwaite model to estimate the monthly potential evapotranspiration in Brazil associated with a Geographic Information System (GIS). To correct the tendency of this model to overestimate $E T_{p}$ when the mean air temperature is higher than $26,{ }^{\circ} \mathrm{C}$, a correction factor was introduced $\left(C_{T}\right)$ simplifying the use of this model in computational operations, such as a Geographic Information System. The result was easily interpreted maps, with great climatological value. 